\title{
Superfluorescent Rayleigh Scattering from Suspensions of Dielectric Particles
}

\author{
G. R. M. Robb and B.W. J. McNeil \\ Department of Physics, John Anderson Building, University of Strathclyde, Glasgow G4 ONG, Scotland
}

(Received 26 August 2002; published 28 March 2003)

\begin{abstract}
We demonstrate that superfluorescent scattering of light can occur when laser light is incident on a collection of dielectric Rayleigh particles suspended in a viscous medium. Using a linear stability analysis, an expression for the spatiotemporal evolution of the scattered (probe) field is derived. An approximate condition for the progression of the interaction into the nonlinear regime is deduced and it is shown that, in the nonlinear regime, the scattered field intensity shows the characteristic quadratic dependence on particle density expected from a superfluorescent or superradiant process, once the effects of pump depletion are accounted for.
\end{abstract}

DOI: 10.1103/PhysRevLett.90.123903

It has long been known that when laser light is incident on dielectric particles that are small compared to the radiation wavelength it undergoes Rayleigh scattering [1]. More recent theoretical work has predicted the existence of a new classical scattering phenomenon in collections of free dielectric Rayleigh particles called "collective Rayleigh scattering" (CRS) [2,3] which involves the spontaneous formation of a particle density modulation on the scale of the radiation wavelength. In the field of nonlinear optics, these density gratings represent novel nonlinear optical media with potential applications as artificial Kerr media [4], tunable photonic band gap materials [5], and as providers of additional feedback in random lasers [6]. The grating may be spontaneously generated via the interaction of the particles with a radiation pump field and a small counterpropagating radiation probe field (which may arise from noise due to random fluctuations in the particle density) that produces periodic ponderomotive forces in the particle ensemble. The collective nature of CRS results in an exponentially growing counterpropagating probe field.

The phenomenon of CRS is directly analogous to the periodic bunching of free electrons in the free-electron laser (FEL) [7], and cold atoms in the collective atomic recoil laser (CARL) [8], both processes resulting in the production of coherently scattered radiation. It has been shown $[9,10]$ that both the FEL and the CARL processes can produce a superfluorescent or superradiant regime, where the scattered (probe) field intensity scales as the square of the electron/atom density, $n$, indicating a coherent scattering process. As this process is initiated by incoherent scattering or noise, we describe it as superfluorescence, as opposed to superradiance, i.e., radiation with an $n^{2}$ dependence from a coherently prepared system, as first described by Dicke [11]. Superfluorescent emission (i.e., superfluorescent Thomson scattering) from an FEL has been demonstrated experimentally in [12] and superfluorescent Rayleigh scattering from a Bose-Einstein condensate was demonstrated in [13]. It was subsequently shown that the latter could be described using a CARL model in the superfluorescent limit [14].
PACS numbers: 42.65.-k, 42.65.Es, 94.10.Gb

In this paper, we show that superfluorescent Rayleigh scattering can occur in a system of Rayleigh microparticles suspended in a viscous medium at room temperature. Under certain conditions, this system can produce highly nonlinear optical behavior, resulting in large amplitude density modulations, large scattered light intensities, and significant pump depletion. Although suspensions of Rayleigh particles have been known to display passive nonlinear optical behavior, e.g., as artificial Kerr media [4] or optical limiters [15], this is the first time that active nonlinear optical behavior (i.e., high gain probe amplification via stimulated scattering) in these systems has been predicted.

The model used to describe the phenomena discussed in this Letter consists of a strong plane pump wave scattered by an initially uniform spatial distribution of dielectric Rayleigh particles suspended in a viscous medium, and an initially very weak counterpropagating plane wave probe, as shown schematically in Fig. 1. In practice, this weak probe field could arise from incoherent spontaneous Rayleigh scattering due to a random particle distribution.

The form of the $\mathbf{E}$-field in the medium is

$$
\mathbf{E}=\mathbf{E}_{1}(z, t)+\mathbf{E}_{2}(z, t)
$$

where $\mathbf{E}_{1}(z, t)=\left[A_{1}(z, t) e^{i(k z-\omega t)}+\right.$ c.c. $] \hat{\mathbf{x}}$ is the electric field of the initially weak probe field and $\mathbf{E}_{2}(z, t)=$ $\left[A_{2}(z, t) e^{-i(k z+\omega t)}+\right.$ c.c. $] \hat{\mathbf{x}}$ is the electric field of the strong pump field, $c / n$ is the speed of light in the (dispersionless) medium, $k_{m}=2 \pi / \lambda_{m}$ is the wave number,

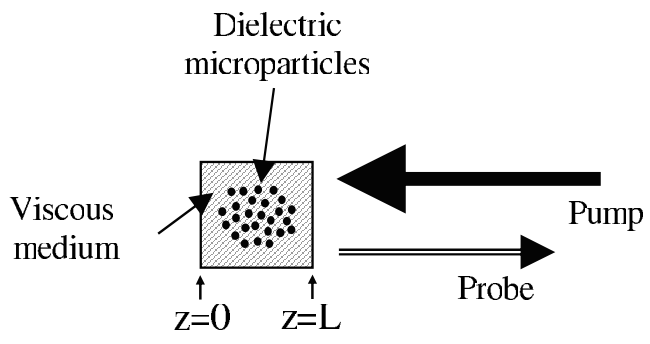

FIG. 1. Schematic diagram of the model. 
$\lambda_{m}=\lambda_{0} / n$ is the wavelength of the light in the medium, $n$ is the refractive index of the medium, and $\hat{\mathbf{x}}$ is a transverse unit vector. For simplicity, we have assumed that the pump and probe are frequency degenerate. Introducing a small detuning between the probe field and the pump does not significantly change the phenomena described in this Letter.

The force on the $j$ th particle exerted by the optical fields can be derived from the Lorentz force equation to be

$$
\mathbf{F}_{j}=\frac{\partial \mathbf{d}_{j}}{\partial t} \times \mathbf{B}\left(z_{j}, t\right),
$$

where $\mathbf{B}(z, t)=(n / c) \hat{\mathbf{z}} \times\left(\mathbf{E}_{1}-\mathbf{E}_{2}\right)$ is the magnetic field of the electromagnetic wave, $\mathbf{d}_{j}$ is the dipole moment of the $j$ th particle induced by the electric field at $z=z_{j}$ (the axial position of the $j$ th particle) given by

$$
\begin{gathered}
\mathbf{d}_{j}=\boldsymbol{\epsilon}_{0} \boldsymbol{\epsilon}_{m} V_{p}\left\{\chi\left[A_{1}\left(z_{j}, t\right) e^{i\left(k z_{j}-\omega t\right)}+A_{2}\left(z_{j}, t\right) e^{-i\left(k z_{j}+\omega t\right)}\right]\right. \\
+ \text { c.c. }\} \hat{\mathbf{x}},
\end{gathered}
$$

where $\epsilon_{0}$ is the permittivity of free space, $\epsilon_{m}$ is the relative permittivity of the viscous medium, $V_{p}=$ $4 \pi a^{3} / 3$ is the particle volume, and $a$ is the particle radius. The susceptibility of the dielectric particle is $\chi=\chi_{1}+$ $i \chi_{2}$, where $\chi_{1}=3\left[\left(\epsilon_{p} / \epsilon_{m}-1\right) /\left(\epsilon_{p} / \epsilon_{m}+2\right)\right]$ and $\chi_{2}=$ $2\left(k_{m} a\right)^{3}\left[\left(\epsilon_{p} / \epsilon_{m}-1\right) /\left(\epsilon_{p} / \epsilon_{m}+2\right)\right]^{2}$ represent the dispersive and dissipative response of the dipole, respectively, and $\epsilon_{p}$ is the relative permittivity of the particle. The dissipative response is due to damping via reradiation, i.e., incoherent Rayleigh scattering. It was shown in [15] that the dynamics of the particles under the influence of the electromagnetic fields, the viscous drag force due to the medium, and the stochastic Brownian forces exerted on the particle by the molecules of the suspending medium can be described by a Fokker-Planck equation describing the evolution of the particle probability distribution. The dipole moments of the moving particles constitute a time-dependent polarization which drives the evolution of the probe and pump radiation fields. The dynamics of the particles and the fields are simultaneously described by a set of coupled Maxwell-FokkerPlanck equations [15]

$$
\begin{aligned}
& \frac{\partial P_{k}(\bar{z}, \bar{t})}{\partial \bar{t}}=i \frac{k}{\bar{\gamma}}\left(\bar{A}_{1} \bar{A}_{2}^{*} P_{k-1}+\bar{A}_{1}^{*} \bar{A}_{2} P_{k+1}\right) \\
& -k\left(\frac{i}{\bar{\gamma}} \alpha\left(\left|\bar{A}_{1}\right|^{2}-\left|\bar{A}_{2}\right|^{2}\right)+\bar{\gamma} \bar{\sigma}^{2}\right) P_{k}, \\
& \left(\frac{\partial}{\partial \bar{t}}+\frac{\partial}{\partial \bar{z}}\right) \bar{A}_{1}(\bar{z}, \bar{t})=(1+i \alpha)\left(2 \pi \bar{A}_{2} P_{1}+i \bar{A}_{1}\right), \\
& \left(\frac{\partial}{\partial \bar{t}}-\frac{\partial}{\partial \bar{z}}\right) \bar{A}_{2}(\bar{z}, \bar{t})=-(1+i \alpha)\left(2 \pi \bar{A}_{1} P_{1}^{*}-i \bar{A}_{2}\right),
\end{aligned}
$$

where $\bar{z}=2 k_{m} \rho z$ and $\bar{t}=2 \omega \rho t$ are scaled position and time variables, $\bar{A}_{1,2}(\bar{z}, \bar{t})=-2 i \sqrt{\left(\epsilon_{0} \epsilon_{m}\right) /\left(\rho N_{p} M c^{2}\right)} A_{1,2}$ are scaled complex probe (1) and pump (2) envelopes respectively, $\bar{\gamma}=\frac{3 \pi a \eta}{\omega \rho M}$ is a scaled viscous damping coefficient, $\bar{\sigma}=\frac{n}{\rho} \sqrt{\left(k_{B} T\right) /\left(M c^{2}\right)}$ is a scaled temperature coefficient, $\alpha \stackrel{\rho}{=} \chi_{2} / \chi_{1}$ is an incoherent scattering coefficient, and $\rho=\left(N_{p} V_{p} \chi_{1}\right) / 4$ is a dimensionless coupling parameter. $P_{k}$ is the $k$ th spatial harmonic of the particle distribution function $P(\bar{z}, \bar{t}, \theta)$; i.e., $P(\bar{z}, \theta, \bar{t})=$ $\sum_{k=-\infty}^{\infty} P_{k}(\bar{z}, \bar{t}) e^{i k \theta}$, where $P_{0}=1 /(2 \pi), P_{-n}=P_{n}^{*}, \theta=$ $2 k_{m} z$, and it has been assumed that the viscous damping is sufficiently strong that inertial effects are negligible, i.e., the particle momentum can be adiabatically eliminated [15]. $k_{B}$ is Boltzmann's constant, $T$ is temperature, $M$ is the mass of each particle, $N_{p}$ is the particle number density, and $\eta$ is the coefficient of viscosity for the medium.

It can be seen from Eqs. (4) and (5) that a particle density modulation $\left(P_{1}\right)$ can couple the pump and probe fields. The nature of the coupling depends on the strength and the phase of the density modulation, i.e., the position in the ponderomotive potential formed by the interference of the pump and probe around which the particles bunch. In what follows, we neglect the effect of the dissipative part of the susceptibility $(\alpha=0)$. This can be achieved by considering sufficiently small particles, as $\alpha \propto\left(k_{m} a\right)^{3}$.

Equations (3)-(5) have a trivial steady-state solution corresponding to a uniform amplitude pump field, no probe field, and uniformly distributed particles; i.e., $\bar{A}_{2}(\bar{z})=\bar{A}_{2_{0}} e^{-i \bar{z}}, \bar{A}_{1}(\bar{z})=0, P_{k}(\bar{z})=0 \forall ; k \neq 0$. We will show that this steady state is unstable to small perturbations in either the probe field or the particle distribution for the case where we can neglect the dissipative response of the dipole moment of the particles $(\alpha \rightarrow 0)$. In this limit, treating the steady-state pump amplitude, $\bar{A}_{2}$, as a zeroth-order quantity and the probe field amplitude, $\bar{A}_{1}$, and particle distribution harmonic amplitudes, $P_{k}$, as first order quantities, the evolution equations are, to first order,

$$
\begin{gathered}
\frac{\partial P_{1}}{\partial \bar{t}}=\frac{i}{\bar{\gamma}}\left(\frac{\bar{A}_{1} \bar{A}_{2_{0}}^{*} e^{i \bar{z}}}{2 \pi}\right)-\bar{\gamma} \bar{\sigma}^{2} P_{1}, \\
\left(\frac{\partial}{\partial \bar{t}}+\frac{\partial}{\partial \bar{z}}\right) \bar{A}_{1}=2 \pi \bar{A}_{2_{0}} e^{-i \bar{z}} P_{1}+i \bar{A}_{1} .
\end{gathered}
$$

Laplace transforming these equations with respect to $\bar{t}$, so that $X(\bar{z}, \bar{t}) \rightarrow \tilde{X}(\bar{z}, s)$, the problem can be reduced to the single linear differential equation,

$$
\frac{\partial \tilde{\bar{A}}_{1}}{\partial \bar{z}}+\left(s-i-\frac{i\left|\bar{A}_{2_{0}}\right|^{2}}{\bar{\gamma}(s+D)}\right) \tilde{\bar{A}}_{1}=\frac{2 \pi \bar{A}_{2_{0}} P_{10} e^{-i \bar{z}}}{s+D},
$$

where $D=\bar{\gamma} \bar{\sigma}^{2}, P_{10}=P_{1}(\bar{z}, \bar{t}=0)$, and we have assumed that $A_{1}(\bar{z}, \bar{t}=0)=0$. Physically, this means that we consider the interaction to start from fluctuations in the particle distribution, rather than an optical seed field. The results would not change significantly if we 
were to consider the opposite case or that where both perturbations were present. Solving (8) with the boundary condition $\tilde{\bar{A}}_{1}(\bar{z}=0)=0$, and taking the inverse Laplace transform of the solution, using $X(\bar{z}, \bar{t})=$ $[1 /(2 \pi i)] \int_{\nu-i \infty}^{\nu+i \infty} \tilde{X}(\bar{z}, s) e^{s \bar{t}} d s$, where $\nu$ is chosen so that the path of integration lies to the right of any singularities in the complex plane, we obtain a solution which can be written as the sum of a term which depends on time only and a term which depends on both space and time:

$$
\bar{A}_{1}(\bar{z}, \bar{t})=\bar{A}_{1}^{(a)}(\bar{t})+\bar{A}_{1}^{(b)}(\bar{z}, \bar{t}),
$$

where

$$
\bar{A}_{1}^{(a)}(\bar{t})=2 \pi \bar{A}_{2_{0}} P_{10}\left(\frac{e^{s_{1} \bar{t}}}{s_{1}-s_{2}}+\frac{e^{s_{2} \bar{t}}}{s_{2}-s_{1}}\right),
$$

$s_{1,2}$ are the two roots of $s(s+D)-i\left[\left(\left|\bar{A}_{2_{0}}\right|^{2}\right) / \bar{\gamma}\right]=0$, and $\bar{A}_{1}^{(b)}(\bar{z}, \bar{t})=-\frac{1}{2 \pi i} \int_{\nu-i \infty}^{\nu+i \infty} \frac{2 \pi \bar{A}_{2_{0}} P_{10}}{\left(s-s_{1}\right)\left(s-s_{2}\right)}$

$$
\times e^{s(\bar{t}-\bar{z})} e^{\left\{\left(i\left|A_{20}\right|^{2} \bar{z}\right) /[\bar{\gamma}(s+D)]\right\}} d s .
$$

The situation for $\bar{t}-\bar{z}>0$ is of most interest and relevance to experiments. It can be seen from inspection of (11) that there will be contributions to the integral from simple poles at $s=s_{1}$ and $s=s_{2}$ and an essential singularity at $s=-D$. Evaluating these contributions, we find

$$
\begin{aligned}
\bar{A}_{1}^{(b)}(\bar{z}, \bar{t})= & -\bar{A}_{1}^{(a)}(\bar{z}) \\
& +i \bar{A}_{2_{0}} P_{10} \oint_{\Gamma} \frac{e^{s(\bar{t}-\bar{z})} e^{\left\{\left(i\left|\bar{A}_{2_{0}}\right| \bar{z}\right) /[\bar{\gamma}(s+D)]\right\}}}{\left(s-s_{1}\right)\left(s-s_{2}\right)} d s,
\end{aligned}
$$

where $\Gamma$ is a counterclockwise path which encircles the essential singularity at $s=-D$, but not the simple poles at $s=s_{1,2}$. Therefore, when $\bar{t}>\bar{z}$,

$$
\bar{A}_{1}(\bar{z}, \bar{t})=i \bar{A}_{2_{0}} P_{10} e^{-D y} \sqrt{x} y \oint_{\Gamma^{\prime}} g(\eta) e^{\sqrt{x y}[\eta+(1 / \eta)]} d \eta,
$$

where we have defined the new variables $x=$ $i\left[\left(\left|\bar{A}_{2_{0}}\right|^{2} \bar{z}\right) / \bar{\gamma}\right], y=\bar{t}-\bar{z}, \eta=\sqrt{y / x}(s+D)$, and defined $g(\eta)=\sqrt{x / y}\left\{1 /\left[\left(\eta-\eta_{1}\right)\left(\eta-\eta_{2}\right)\right]\right\}$. Note that the path of integration $\Gamma^{\prime}$ encircles the essential singularity at $\eta=0$ but not $\eta_{1,2}=\sqrt{y / x}\left(s_{1,2}+D\right)$. Applying the method of stationary phase to (13) produces the asymptotic result

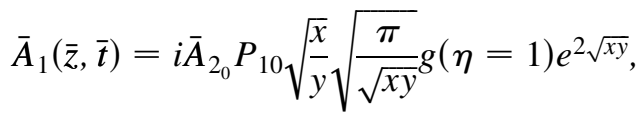

when $|\sqrt{x y}| \gg 1$. Consequently, the scaled probe intensity $\left|\bar{A}_{1}\right|^{2}$ grows as

$$
\left|\bar{A}_{1}(\bar{z}, \bar{t})\right|^{2} \propto\left|P_{10}\right|^{2} e^{-D(\bar{t}-\bar{z})} e^{2 \sqrt{2} \sqrt{\left[\left|\bar{A}_{2_{0}}\right|^{2} \overline{\bar{z}}(\bar{t}-\bar{z})\right] / \bar{\gamma}}} .
$$

Note that the exponentially decaying term will cause saturation of the probe intensity in the linear regime when

$$
\bar{t}=\bar{t}_{\text {lin }} \equiv \frac{8\left|\bar{A}_{2_{0}}\right|^{2} \bar{L}}{\bar{\gamma}^{3} \bar{\sigma}^{4}}
$$

where we have assumed $\bar{z}=\bar{L}$ and $\bar{t} \gg \bar{L}$. If this "linear saturation time" is very large, then the probe intensity and particle density modulation amplitude $\left|P_{1}\right|$ will be sufficiently large that linear theory breaks down and nonlinear effects must be taken into account. Saturation in this case will occur due to nonlinear phenomena such as pump depletion and generation of higher spatial harmonics of the particle density distribution. In order to obtain an estimate of the time scale at which nonlinear effects become important, we make use of the fact that the density modulation amplitude, $\left|P_{1}\right|$, cannot increase indefinitely, but has a maximum value of $1 /(2 \pi)$, corresponding to a perfectly bunched collection of particles at points separated by $\lambda_{m} / 2$. Consequently, since in the linear regime $P_{1}$ will have the same dependence on $\bar{t}$ as $\bar{A}_{1}$ in Eq. (15), i.e., $\left|P_{1}\right| \approx\left|P_{10}\right| \exp \left[\sqrt{\left(2\left|\bar{A}_{2_{0}}\right|^{2} \bar{L} \bar{t}\right) / \bar{\gamma}}\right]$ when $\bar{z}=\bar{L}$ and $\bar{t} \gg \bar{z}$, where we have neglected the exponentially decaying term in Eq. (15), then nonlinear effects will become important when $\left|P_{1}\right| \sim \frac{1}{2 \pi}$ or when $\bar{t} \sim \bar{t}_{\mathrm{nl}}$, where

$$
\bar{t}_{\mathrm{nl}} \equiv \frac{\bar{\gamma}}{2\left|\bar{A}_{2_{0}}\right|^{2} \bar{L}}\left[\ln \left(\frac{1}{2 \pi\left|P_{10}\right|}\right)\right]^{2} .
$$

In this Letter, we are most interested in the nonlinear regime where the particle density modulation and probe intensity become large. We therefore require that $\bar{t}_{\text {nl }}<\bar{t}_{\text {lin }}$. This inequality can be expressed as a threshold condition for the scaled pump intensity using Eqs. (16) and (17), which must satisfy

$$
\left|\bar{A}_{2_{0}}\right|^{2}>\frac{\bar{\gamma}^{2} \bar{\sigma}^{2}}{4 \bar{L}^{2}} \ln \left(\frac{1}{2 \pi\left|P_{10}\right|}\right),
$$

in order that the interaction can reach the nonlinear regime where large density modulations and probe intensities can occur.

In order to observe the evolution of the instability predicted by the linear analysis into the nonlinear regime, we use a numerical solution of equations (3)-(5). The number of spatial harmonics $k_{\max }$ used at each point $\bar{z}$ is chosen to be sufficiently large that the solution is unaffected by further increasing $k_{\max }$. The boundary conditions used are $\bar{A}_{1}(\bar{z}=0, \bar{t})=0, \bar{A}_{2}(\bar{z}=\bar{L}, \bar{t})=$ $\bar{A}_{2_{0}}$, and $P_{1}(\bar{z}=0, \bar{t})=P_{1_{0}} e^{i \xi(\bar{z})}$, and the initial conditions are $\bar{A}_{1}(\bar{z}, \bar{t}=0)=0, \quad \bar{A}_{2}(\bar{z}, \bar{t}=0)=\bar{A}_{2_{0}}$, and $P_{1}(\bar{z}, \bar{t}=0)=P_{1_{0}} e^{i \xi(\bar{z})}$, where $\xi(\bar{z})$ is a random phase. Figure 2 shows a graph of probe intensity as a function of $\bar{z}$ and $\bar{t}$ as calculated from Eqs. (3) -(5) using the parameters $\bar{L}=0.45,\left|P_{10}\right|=1 \times 10^{-4}, \bar{A}_{2}=0.1, \bar{\gamma}=1$, and $\bar{\sigma}=2.6 \times 10^{-3}$. These correspond to the real physical parameters $\lambda=532 \mathrm{~nm}, N_{p}=10^{11} \mathrm{~cm}^{-3}, L=3 \mathrm{~cm}$, $a=25 \mathrm{~nm}, \epsilon_{m}=1.769, \epsilon=2.56, \quad T=300 \mathrm{~K}, \quad$ and pump intensity $I_{2}=3.82 \times 10^{10} \mathrm{~W} \mathrm{~cm}^{2}$, and have been chosen so that the scaled pump intensity easily satisfies 


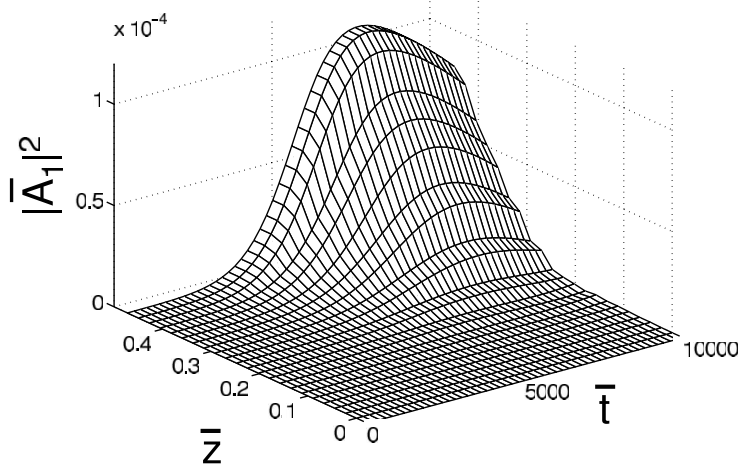

FIG. 2. Spatiotemporal evolution of scaled probe intensity $\left|\bar{A}_{1}(\bar{z}, \bar{t})\right|^{2}$.

the condition (18) and the incoherent scattering parameter $\alpha$ is small $(\alpha=0.0056)$. These parameters correspond physically to a suspension of small latex microspheres in water and are mostly similar to the experiment of [15]. The main exception is the pump intensity, which is significantly smaller and $\mathrm{cw}$ in [15]. The larger pump laser intensity required to observe superfluorescent Rayleigh scattering as in Fig. 2 will require a pulsed laser to be used, with a pulse duration $\geq 2 \mu \mathrm{s}$. It can be seen from Fig. 2 that the scaled probe intensity is strongly amplified until it saturates at a value around $1.4 \%$ of the pump intensity. The amplification of the probe is simultaneous with the amplification of $\left|P_{1}\right|$. Therefore the probe amplification is due to the spontaneous formation of a periodic density modulation of the particle density with spatial period $\lambda_{m} / 2$. Saturation in this case is mainly due to the excitation of higher spatial harmonics of the particle density ( $k_{\max } \approx 50$ for this example) although pump depletion is another possible saturation mechanism in this nonlinear regime.

Figure 3 shows a graph of peak probe intensity against $\rho$, i.e., against $N_{p}$, with all other real parameters as for Fig. 2. It can be seen that peak scaled probe intensity decreases with increasing $\rho$. This decrease in scaled probe intensity is due to depletion of the pump field. When the evolution of the pump field is artificially switched off, it can be seen that the peak scaled probe intensity remains almost constant, which implies that the real probe intensity obeys $I_{1_{\text {peak }}} \propto N_{p}^{2}$, so the peak intensity of the probe field approximately shows the $N_{p}^{2}$ dependence characteristic of a superfluorescent or superradiant process.

In conclusion, it has been shown that superfluorescent scattering of light can occur at room temperature when laser light is incident on a collection of dielectric particles suspended in a viscous medium. The results presented in this Letter suggest that suspensions of dielectric Rayleigh particles are extremely interesting and potentially useful optical media as they can display collective scattering

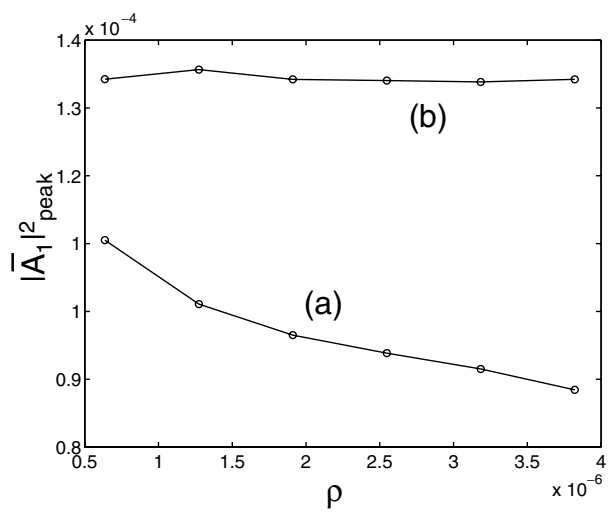

FIG. 3. Graph of peak scaled probe intensity $\left|\bar{A}_{1}\right|_{\text {peak }}^{2}$ against $\rho$ when (a) pump field is allowed to evolve self-consistently and (b) when pump field is held constant.

phenomena arising from particle center-of-mass motion without, as in the case of atomic vapors, requiring ultracold temperatures.

The authors thank the Engineering and Physical Sciences Research Council for support via research Grant No. GR/N32686.

[1] H. C. van de Hulst, Light Scattering by Small Particles (Dover, New York, 1981).

[2] B.W. J. McNeil and G. R. M. Robb, Opt. Commun. 148, 54 (1998).

[3] S. M. Wiggins, G. R. M. Robb, B.W. J. McNeil, D. R. Jones, S. P. Jamison, and D. A. Jaroszynski, Meas. Sci. Technol. 13, 263 (2002).

[4] P.W. Smith, P. J. Maloney, and A. Ashkin, Opt. Lett. 7, 347 (1982).

[5] A. Moroz, Europhys. Lett. 50, 466 (2000).

[6] H. Cao, Y. G. Zhao, S. T. Ho, E.W. Seelig, Q. H. Wang, and R. P. H. Chang, Phys. Rev. Lett. 82, 2278 (1999).

[7] E. L. Saldin, E. A. Schneidmiller, and M.V. Yurkov, The Physics of Free-Electron Lasers (Springer-Verlag, Berlin, 2000); R. Bonifacio et al., Riv. Nuovo Cimento 9, 1 (1990).

[8] R. Bonifacio, L. De Salvo, L. M. Narducci, and E. J. D’Angelo, Phys. Rev. A 50, 1716 (1994).

[9] R. Bonifacio and F. Casagrande, Lett. Nuovo Cimento 37, 39 (1983).

[10] R. Bonifacio and L. De Salvo, Appl. Phys. B 60, S233 (1995).

[11] R. H. Dicke, Phys. Rev. 93, 99 (1954).

[12] D. A. Jaroszynski et al., Phys. Rev. Lett. 78, 1699 (1997).

[13] S. Inouye, A. P. Chikkatur, D. M. Stamper-Kurn, J. Stenger, D. E. Pritchard, and W. Ketterle, Science 285, 571 (1999).

[14] N. Piovella, M. Gatelli, L. Martinucci, R. Bonifacio, B.W. J. McNeil, and G. R. M. Robb, Laser Phys. 12, 188 (2002).

[15] S. M. Wiggins, G. R. M. Robb, B.W. J. McNeil, D. R. Jones, S. P. Jamison, and D. A. Jaroszynski, J. Mod. Opt. 49, 997 (2002). 